\title{
PARABOLIC EQUATIONS AND THERMODYNAMICS
}

\author{
BY
}

W. A. DAY

\section{Hertford College, Oxford, England}

1. Introduction. The aim of this paper is to prove two theorems about periodic solutions of parabolic equations which are suggested by considerations drawn from thermodynamics and, in particular, by two papers of Clausius. (These papers are readily accessible as items 7 and 8 in the collection [1], edited by Kestin. For the history of thermodynamics up to 1854 see Truesdell [2], and for comments on the interpretations to be assigned to certain verbal statements of a second law see Serrin [3] and Day [4, 5].)

We are concerned with solutions $u(x, t)$ of the parabolic equation

$$
\left(a(x) u_{x}\right)_{x}=b(x) u_{t} \quad(0<x<1,-\infty<t<+\infty)
$$

which satisfy boundary conditions of the form

$$
u(0, t)=u(1, t)=\tau(t) \quad(-\infty<t<+\infty) .
$$

Both $u$ and $\tau$ are understood to be periodic, with period $p$, in their dependence upon $t$, i.e., $u(x, t+p)=u(x, t)$ and $\tau(t+p)=\tau(t)$, and it is assumed that the coefficients $a$ and $b$ are positive on $[0,1]$ and that $a \in C^{1}, b \in C^{0}$, and $\tau \in C^{3}$.

The unique periodic $u$ corresponding to a given periodic $\tau$, with Fourier expansion

$$
\tau(t)=\sum_{-\infty}^{+\infty} c_{n} \exp \left(\frac{2 \pi i n t}{p}\right)
$$

can be constructed as the sum

$$
u(x, t)=\sum_{-\infty}^{+\infty} c_{n} U_{n}(x) \exp \left(\frac{2 \pi i n t}{p}\right)
$$

in which the complex-valued $U_{n}$ are the solutions of the boundary value problems

$$
\left(a(x) U_{n}^{\prime}\right)^{\prime}=\frac{2 \pi i n}{p} b(x) U_{n}, \quad U_{n}(0)=U_{n}(1)=1 .
$$

(The primes denote derivatives with respect to $x$.)

We may think of $u$ as the temperature in a rigid slab whose faces $x=0$ and $x=1$ are in contact with a heat reservoir, and which performs cycles of period Received January 7, 1991. 
$p$. The reservoir temperature $\tau$ varies periodically with the time $t$ but is spatially homogeneous at each instant. If, as we suppose in this section and the next, $u$ and $\tau$ are measured on an absolute scale then each will be positive. The coefficients $a$ and $b$ are, respectively, the thermal conductivity and the heat capacity of the slab.

The rate at which the slab loses heat to the reservoir by conduction across the face $x=0$ is $a(0) u_{x}(0, t)$ and the rate at which the slab gains heat from the reservoir by conduction across the face $x=1$ is $a(1) u_{x}(1, t)$. The difference

$$
Q(t)=a(1) u_{x}(1, t)-a(0) u_{x}(0, t)
$$

is thus the net rate-of-heating of the slab.

If $\tau$ is nonconstant, the cycles performed by the slab are irreversible, and thermodynamic arguments suggest that the strict inequality

$$
\int_{0}^{p} \frac{Q}{\tau} d t<0
$$

ought to be valid. On the other hand, if $\tau$ varies sufficiently slowly, the cycles are nearly reversible and the equation

$$
\int_{0}^{p} \frac{Q}{\tau} d t=0
$$

ought to be approximately true.

These conclusions are due to Clausius and we shall refer to (1.4) as the Clausius inequality; it should be noted, though, that our notation differs from that of Clausius, who did not make explicit the presence of the time as an independent variable.

We shall verify, to begin with, that the inequality (1.4) holds and that (1.5) holds approximately if $\tau$ varies sufficiently slowly. We shall go on to show-and this is the main conclusion of Theorem 1-that, to a more accurate approximation, an equation

$$
\int_{0}^{p} \frac{Q}{\tau} d t+A \int_{0}^{p}\left(\frac{Q}{\tau}\right)^{2} d t=0
$$

holds, where $A$ is a positive constant depending only upon the coefficients $a$ and $b$. This last conclusion appears to be new. It would be of considerable interest to ascertain whether such strengthened versions of the Clausius inequality hold within theories which permit the slab to deform.

The slab absorbs heat from the reservoir at any instant $t$ at which $Q(t)>0$, and it emits heat to the reservoir at any instant $t$ at which $Q(t)<0$; thus the maximum temperature at which heat is absorbed is

$$
T^{+}=\sup \{\tau(t):-\infty<t<+\infty \text { and } Q(t)>0\},
$$

and the minimum temperature at which heat is emitted is

$$
T^{-}=\inf \{\tau(t):-\infty<t<+\infty \text { and } Q(t)<0\} .
$$

In the present context it can be shown (see the proof of Theorem 2) that the integral

$$
\int_{0}^{p} Q d t=0
$$


and it is a simple matter to deduce from (1.9) and (1.4) that

$$
T^{+}>T^{-} \text {. }
$$

This last inequality tells us that it is not possible for there to be a temperature $T_{0}$ such that $\tau(t)<T_{0}$ for every $t$ for which $Q(t)>0$, and $\tau(t)>T_{0}$ for every $t$ for which $Q(t)<0$. Thus, in cyclic processes, it is not possible for the slab to absorb heat and then emit it at higher temperatures.

Such a conclusion would seem to be in line with Clausius' statement of a second law which says that "it is impossible to devise an engine which, working in a cycle, shall produce no effect other than the transfer of heat from a colder to a hotter body."

Our Theorem 2 improves upon (1.10) by providing a quantitative lower bound, in terms of the reservoir temperature $\tau$, on the separation between $T^{+}$and $T^{-}$. Once again it would be of interest to ascertain whether such a conclusion can be extended to theories which permit the slab to deform.

2. The Clausius inequality and slowly-varying solutions. In what follows it is convenient to introduce a small positive parameter $\varepsilon$ which measures the slowness with which the reservoir temperature varies over a long period. More exactly, we shall say that $\tau$ is slowly-varying if there are positive constants $m, m_{0}, M, M_{k}$ $(k=0,1,2,3)$, independent of $\varepsilon$, such that

$$
\begin{gathered}
m_{0} \varepsilon^{-1} \leq p \leq M_{0} \varepsilon^{-1}, \quad m \leq \tau \leq M, \\
\left|\tau^{(k)}\right| \leq M_{k} \varepsilon^{k} \quad(k=1,2,3),
\end{gathered}
$$

where $\tau^{(k)}$ is the derivative of order $k$ with respect to $t$. It should be noted that this definition forces $\tau$ to be positive and bounded away from zero.

The conditions (2.1) are met if, for example,

$$
\tau(t)=\tau_{0}(\varepsilon t) \quad(-\infty<t<+\infty),
$$

where $\tau_{0}$ is $C^{3}$, positive, and periodic, with period $p_{0}$ say, and is independent of $\varepsilon ;$ in this case $\tau$ has periodic $p=p_{0} \varepsilon^{-1}$.

We use the notation $\alpha=O\left(\varepsilon^{n}\right)$ to mean that there is a positive constant $\mu$, depending only on the coefficients $a$ and $b$ and the constants $m, m_{0}, M, M_{k}$ $(k=0,1,2,3)$, such that $|\alpha| \leq \mu \varepsilon^{n}$ for all sufficiently small values of the parameter $\varepsilon$.

We introduce the positive constant

$$
A=\int_{0}^{1} b v d x /\left(\int_{0}^{1} b d x\right)^{2},
$$

$v$ being the solution of the boundary value problem

$$
\left(a v^{\prime}\right)^{\prime}=-b, \quad v(0)=v(1)=0,
$$

and turn to our first theorem. 
THEOREM 1. The Clausius inequality holds whenever $u$ and $\tau$ are positive and $\tau$ is nonconstant. If $\tau$ is slowly-varying then the order relation

$$
\int_{0}^{p} \frac{Q}{\tau} d t=O(\varepsilon)
$$

holds, and so does the more accurate order relation

$$
\int_{0}^{p} \frac{Q}{\tau} d t+A \int_{0}^{p}\left(\frac{Q}{\tau}\right)^{2} d t=O\left(\varepsilon^{2}\right) .
$$

The proof of the Clausius inequality is straightforward. For, if $u$ is positive, equation (1.1) tells us that

$$
\left(\frac{a u_{x}}{u}\right)_{x}=-\frac{a u_{x}^{2}}{u^{2}}+\frac{b u_{t}}{u}
$$

Thus, if we integrate with respect to $x$, and note that, as (1.2) and (1.3) imply,

$$
\int_{0}^{1}\left(\frac{a u_{x}}{u}\right)_{x} d x=\frac{a(1) u_{x}(1, t)}{u(1, t)}-\frac{a(0) u_{x}(0, t)}{u(0, t)}=\frac{Q}{\tau}
$$

we deduce that

$$
\frac{Q}{\tau}+\int_{0}^{1} \frac{a u_{x}^{2}}{u^{2}} d x=\frac{d}{d t} \int_{0}^{1} b \log u d x .
$$

A further integration with respect to $t$ and an appeal to periodicity now yield the equation

$$
\int_{0}^{p} \frac{Q}{\tau} d t+\int_{0}^{p} \int_{0}^{1} \frac{a u_{x}^{2}}{u^{2}} d x d t=0
$$

and so the inequality

$$
\int_{0}^{p} \frac{Q}{\tau} d t \leq 0
$$

must hold. To see that, as (1.4) asserts, the integral must actually be negative, suppose, to the contrary, that the integral were to vanish. According to (2.6) this can happen only if $u_{x} \equiv 0$ throughout the strip $[0,1] \times(-\infty,+\infty)$ and so, as the boundary conditions (1.2) imply, only if $u \equiv \tau$ throughout the strip. The differential equation (1.1) would then lead us to conclude that $\tau$ is constant; thus the strict inequality (1.4) must hold whenever $\tau$ is nonconstant.

In order to verify the order relations (2.4) and (2.5) we start by defining a function $w$ by way of the equation

$$
u(x, t)=\tau(t)-v(x) \tau^{(1)}(t)+w(x, t) .
$$

This definition forces $w$ to be periodic in $t$; moreover, it follows from (1.1), (1.2), (2.3), and (2.7), that $w$ must satisfy the equation

$$
\left(a w_{x}\right)_{x}=b w_{t}-b v \tau^{(2)}
$$

and the boundary conditions

$$
w(0, t)=w(1, t)=0 \quad(-\infty<t<+\infty) .
$$


According to (1.3) and (1.1), the net rate-of-heating

$$
Q=\int_{0}^{1}\left(a u_{x}\right)_{x} d x=\int_{0}^{1} b u_{t} d x
$$

and if we substitute for $u$ from (2.7) we see that

$$
Q=\left(\int_{0}^{1} b d x\right) \tau^{(1)}-\left(\int_{0}^{1} b v d x\right) \tau^{(2)}+q,
$$

where

$$
q=\int_{0}^{1} b w_{t} d x
$$

Hence

$$
\frac{Q}{\tau}=\left(\int_{0}^{1} b d x\right) \frac{d}{d t} \log \tau-\left(\int_{0}^{1} b v d x\right) \frac{\tau^{(2)}}{\tau}+\frac{q}{\tau},
$$

and, on integrating with respect to $t$ and invoking the periodicity of $\tau$, we see that

$$
\int_{0}^{p} \frac{Q}{\tau} d t=\alpha
$$

where

$$
\alpha=-\left(\int_{0}^{1} b v d x\right) \int_{0}^{p} \frac{\tau^{(2)}}{\tau} d t+\int_{0}^{p} \frac{q}{\tau} d t .
$$

If we now appeal to the restrictions (2.1), which define what it means for $\tau$ to be slowly-varying, we see that

$$
\begin{aligned}
|\alpha| & \leq O(\varepsilon)+O(1) \int_{0}^{p}|q| d t \leq O(\varepsilon)+O(1)\left(p \int_{0}^{p} q^{2} d t\right)^{1 / 2} \\
& =O(\varepsilon)+O\left(\varepsilon^{-1 / 2}\right)\left(\int_{0}^{p} q^{2} d t\right)^{1 / 2} .
\end{aligned}
$$

At a later stage we shall show that

$$
\int_{0}^{p} q^{2} d t=O\left(\varepsilon^{5}\right)
$$

and it will then follow immediately from (2.15) and (2.16) that $\alpha=O(\varepsilon)$. A glance at (2.14) now shows us that the order relation (2.4) is correct.

On the other hand, if the constant $A$ is defined as in equation (2.2) then, after a little calculation, we find that (2.13) implies the equation

$$
\begin{aligned}
\frac{Q}{\tau}+A\left(\frac{Q}{\tau}\right)^{2}= & \left(\int_{0}^{1} b d x\right) \frac{d}{d t} \log \tau-\left(\int_{0}^{1} b v d x\right) \frac{d^{2}}{d t^{2}} \log \tau \\
& -2 A \int_{0}^{1} b d x \cdot \int_{0}^{1} b v d x \cdot \frac{\tau^{(1)} \tau^{(2)}}{\tau^{2}}+A\left(\int_{0}^{1} b v d x\right)^{2}\left(\frac{\tau^{(2)}}{\tau}\right)^{2} \\
& +\left(1+2 A \int_{0}^{1} b d x \cdot \frac{\tau^{(1)}}{\tau}-2 A \int_{0}^{1} b v d x \cdot \frac{\tau^{(2)}}{\tau}\right) \frac{q}{\tau}+A\left(\frac{q}{\tau}\right)^{2}
\end{aligned}
$$


Thus, by virtue of periodicity,

$$
\int_{0}^{p} \frac{Q}{\tau} d t+A \int_{0}^{p}\left(\frac{Q}{\tau}\right)^{2} d t=\beta
$$

where

$$
\begin{aligned}
\beta= & -2 A \int_{0}^{1} b d x \cdot \int_{0}^{1} b v d x \cdot \int_{0}^{p} \frac{\tau^{(1)} \tau^{(2)}}{\tau^{2}} d t+A\left(\int_{0}^{1} b v d x\right)^{2} \int_{0}^{p}\left(\frac{\tau^{(2)}}{\tau}\right)^{2} d t \\
& +\int_{0}^{p}\left(1+2 A \int_{0}^{1} b d x \cdot \frac{\tau^{(1)}}{\tau}-2 A \int_{0}^{1} b v d x \cdot \frac{\tau^{(2)}}{\tau}\right) \frac{q}{\tau} d t+A \int_{0}^{p}\left(\frac{q}{\tau}\right)^{2} d t .
\end{aligned}
$$

On using the restrictions (2.1) to estimate the right-hand side we see that

$$
\begin{aligned}
|\beta| & \leq O\left(\varepsilon^{2}\right)+O\left(\varepsilon^{3}\right)+O(1) \int_{0}^{p}|q| d t+O(1) \int_{0}^{p} q^{2} d t \\
& \leq O\left(\varepsilon^{2}\right)+O(1)\left(p \int_{0}^{p} q^{2} d t\right)^{1 / 2}+O(1) \int_{0}^{p} q^{2} d t \\
& =O\left(\varepsilon^{2}\right)+O\left(\varepsilon^{-1 / 2}\right)\left(\int_{0}^{p} q^{2} d t\right)^{1 / 2}+O(1) \int_{0}^{p} q^{2} d t .
\end{aligned}
$$

Thus, once the order relation (2.16) has been established we shall be able to conclude that $\beta=O\left(\varepsilon^{2}\right)$ and then the order relation (2.5) will follow immediately from (2.17).

To prove (2.16) let $\lambda$ be the least eigenvalue, and let $\psi$ be a corresponding eigenfunction, for the Sturm-Liouville problem

$$
\left(a(x) \psi^{\prime}\right)^{\prime}=-\lambda b(x) \psi, \quad \psi(0)=\psi(1)=0 .
$$

As is well known, $\lambda$ is positive and $\psi$ has no zero in the open interval $(0,1)$; thus we may suppose $\psi$ to be positive in $(0,1)$.

Next, we multiply equation (2.8) through by $w$, integrate by parts, and appeal to the boundary conditions $(2.9)$, to find that

$$
\int_{0}^{1} a w_{x}^{2} d x+\frac{1}{2} \frac{d}{d t} \int_{0}^{1} b w^{2} d x=\tau^{(2)} \int_{0}^{1} b v w d x .
$$

Thus if we integrate with respect to $t$, and remember that $w$ is periodic, we have

$$
\int_{0}^{p} \int_{0}^{1} a w_{x}^{2} d x d t=\int_{0}^{p} \int_{0}^{1} b v w \tau^{(2)} d x d t
$$

On estimating the right-hand side with the aid of the Cauchy-Schwarz inequality we see that

$$
\left(\int_{0}^{p} \int_{0}^{1} b v w \tau^{(2)} d x d t\right)^{2} \leq \int_{0}^{1} b v^{2} d x \cdot \int_{0}^{p}\left|\tau^{(2)}\right|^{2} d t \cdot \int_{0}^{p} \int_{0}^{1} b w^{2} d x d t .
$$

On the other hand, the boundary conditions (2.9) and the variational characterization of the eigenvalue $\lambda$ ensure that

$$
\lambda \int_{0}^{1} b w^{2} d x \leq \int_{0}^{1} a w_{x}^{2} d x,
$$


and when we combine (2.19), (2.20), and (2.21), we see that

$$
\int_{0}^{p} \int_{0}^{1} b w^{2} d x d t \leq \frac{1}{\lambda^{2}} \int_{0}^{1} b v^{2} d x \cdot \int_{0}^{p}\left|\tau^{(2)}\right|^{2} d t
$$

Likewise, if we differentiate (2.8) and (2.9) with respect to $t$ and repeat this argument, but with $w$ replaced by $w_{t}$ and $\tau$ replaced by $\tau^{(1)}$, we conclude that

$$
\int_{0}^{p} \int_{0}^{1} b w_{t}^{2} d x d t \leq \frac{1}{\lambda^{2}} \int_{0}^{1} b v^{2} d x \int_{0}^{p}\left|\tau^{(3)}\right|^{2} d t=O\left(\varepsilon^{5}\right) .
$$

Hence

$$
\int_{0}^{p} q^{2} d t=\int_{0}^{p}\left(\int_{0}^{1} b w_{t} d x\right)^{2} d t \leq \int_{0}^{1} b d x \int_{0}^{p} \int_{0}^{1} b w_{t}^{2} d x d t=O\left(\varepsilon^{5}\right)
$$

which is (2.16), and so the proof of Theorem 1 is complete.

3. An estimate for $T^{+}-T^{-}$. Our second theorem provides an estimate upon $T^{+}-T^{-}$in terms of the reservoir temperature $\tau$ and the properties of the slab; the estimate improves upon (1.10). It is no longer necessary to suppose $u$ and $\tau$ to be positive and $\tau$ need not be slowly-varying.

In what follows the mean reservoir temperature is

$$
\bar{\tau}=\frac{1}{p} \int_{0}^{p} \tau d t
$$

and the $L^{2}$-norm of a function $f$, of period $p$, is

$$
\|f\|=\left(\frac{1}{p} \int_{0}^{p} f^{2} d t\right)^{1 / 2}
$$

THEOREM 2. There is a positive constant $B$, depending only upon the period $p$ and the coefficients $a$ and $b$, such that

$$
T^{+}-T^{-} \geq B \frac{\|\tau-\bar{\tau}\|^{2}}{\left\|\tau^{(1)}\right\|}
$$

whenever $\tau$ is nonconstant.

What we shall show is that (3.1) holds with

$$
B=\frac{2 \lambda}{1+\frac{\lambda^{2} p^{2}}{4 \pi^{2}}} \cdot \frac{\left(\int_{0}^{1} b \psi d x\right)^{2}}{\int_{0}^{1} b d x \int_{0}^{1} b \psi^{2} d x},
$$

where, as in Sec. $2, \lambda$ is the least eigenvalue of the Sturm-Liouville problem (2.18), and $\psi$ is a corresponding eigenfunction.

The first step in the proof is to verify that equation (1.9) is valid in the present circumstances. This is straightforward, since definition (1.3) and the differential equation (1.1) imply that the net rate-of-heating

$$
Q=\int_{0}^{1}\left(a u_{x}\right)_{x} d x=\int_{0}^{1} b u_{t} d x=\frac{d}{d t} \int_{0}^{1} b u d x
$$


Thus periodicity implies, as required, that $\int_{0}^{p} Q d t=0$.

Next, we multiply both sides of the differential equation (1.1) by $u$ and then rearrange to arrive at the equation

$$
\left(u a u_{x}\right)_{x}=a u_{x}^{2}+b u u_{t} .
$$

On integrating with respect to $x$, and noting that, as (1.3) and the boundary conditions (1.2) tell us,

$$
\int_{0}^{1}\left(u a u_{x}\right)_{x} d x=u(1, t) a(1) u_{x}(1, t)-u(0, t) a(0) u_{x}(0, t)=\tau Q
$$

we see that

$$
\tau Q=\int_{0}^{1} a u_{x}^{2} d x+\frac{1}{2} \frac{d}{d t} \int_{0}^{1} b u^{2} d x .
$$

By means of an integration with respect to $t$, and a further appeal to periodicity, we obtain the equation

$$
\int_{0}^{p} \tau Q d t=\int_{0}^{p} \int_{0}^{1} a u_{x}^{2} d x d t
$$

We now appeal to (1.9) and the definitions (1.7) and (1.8) and argue that

$$
\begin{aligned}
\int_{0}^{p} \tau Q d t & =\frac{1}{2} \int_{0}^{p} \tau(|Q|+Q) d t-\frac{1}{2} \int_{0}^{p} \tau(|Q|-Q) d t \\
& \leq \frac{1}{2} T^{+} \int_{0}^{p}(|Q|+Q) d t-\frac{1}{2} T^{-} \int_{0}^{p}(|Q|-Q) d t \\
& =\frac{1}{2}\left(T^{+}-T^{-}\right) \int_{0}^{p}|Q| d t .
\end{aligned}
$$

On invoking the Cauchy-Schwarz inequality in the form

$$
\int_{0}^{p}|Q| d t \leq\left(p \int_{0}^{p} Q^{2} d t\right)^{1 / 2}=p\|Q\|
$$

we deduce from (3.3) and (3.4) that

$$
T^{+}-T^{-} \geq \frac{1}{p} \int_{0}^{p} \int_{0}^{1} a u_{x}^{2} d x d t /\|Q\|
$$

If we can prove the estimates

$$
\begin{gathered}
\|Q\| \leq \int_{0}^{1} b d x\left\|\tau^{(1)}\right\|, \\
\int_{0}^{p} \int_{0}^{1} a u_{x}^{2} d x d t \geq \frac{\lambda_{p}}{1+\frac{\lambda^{2} p^{2}}{4 \pi^{2}}} \cdot \frac{\left(\int_{0}^{1} b \psi d x\right)^{2}}{\int_{0}^{1} b \psi^{2} d x}\|\tau-\bar{\tau}\|^{2},
\end{gathered}
$$

then (3.1) will follow from (3.5), (3.6), and (3.7).

In order to verify (3.6) let

$$
I(x)=\int_{0}^{p}[u(x, t)]^{2} d t .
$$


Then

$$
\begin{gathered}
I^{\prime}=2 \int_{0}^{p} u u_{x} d t \\
\left(a I^{\prime}\right)^{\prime}=2 \int_{0}^{p}\left(u a u_{x}\right)_{x} d t=2 \int_{0}^{p}\left(a u_{x}^{2}+u\left(a u_{x}\right)_{x}\right) d t
\end{gathered}
$$

and if we appeal to the differential equation (1.1) and to the periodicity of $u$ we see that

$$
\left(a I^{\prime}\right)^{\prime}=2 \int_{0}^{p}\left(a u_{x}^{2}+b u u_{t}\right) d t=2 a \int_{0}^{p} u_{x}^{2} d t+b \int_{0}^{p}\left(u^{2}\right)_{t} d t=2 a \int_{0}^{p} u_{x}^{2} d t
$$

Thus $I$ satisfies the differential inequality $\left(a I^{\prime}\right)^{\prime} \geq 0$, and, since the boundary conditions (1.2) imply that

$$
I(0)=I(1)=\int_{0}^{p} \tau^{2} d t
$$

a standard argument assures us that

$$
I(x) \leq \int_{0}^{p} \tau^{2} d t \quad(0 \leq x \leq 1)
$$

i.e.,

$$
\int_{0}^{p}[u(x, t)]^{2} d t \leq \int_{0}^{p} \tau^{2} d t \quad(0 \leq x \leq 1) .
$$

Furthermore, if we differentiate both sides of the equation (1.1) with respect to $t$ and repeat this argument, but with $u$ replaced by $u_{t}$ and $\tau$ replaced by $\tau^{(1)}$, we deduce the inequality

$$
\int_{0}^{p}\left[u_{t}(x, t)\right]^{2} d t \leq \int_{0}^{p}\left[\tau^{(1)}\right]^{2} d t \quad(0 \leq x \leq 1)
$$

Thus, if we recall that

$$
Q=\int_{0}^{1} b u_{t} d x
$$

and invoke the Cauchy-Schwarz inequality again we deduce that

$$
Q^{2} \leq \int_{0}^{1} b d x \int_{0}^{1} b u_{t}^{2} d x
$$

and, therefore, that

$$
\begin{aligned}
\|Q\|^{2} & =\frac{1}{p} \int_{0}^{p} Q^{2} d t \leq \int_{0}^{1} b d x \cdot \frac{1}{p} \int_{0}^{p} \int_{0}^{1} b u_{t}^{2} d x d t \\
& =\int_{0}^{1} b d x \cdot \int_{0}^{1} b\left(\frac{1}{p} \int_{0}^{p} u_{t}^{2} d t\right) d x \\
& \leq\left(\int_{0}^{1} b d x\right)^{2}\left\|\tau^{(2)}\right\|^{2}
\end{aligned}
$$

which proves (3.6). 
In order to prove (3.7) we put

$$
u(x, t)=\phi(x, t)+\tau(t) .
$$

Then we see from (1.1) and (1.2) that $\phi$ must satisfy the differential equation

$$
\left(a(x) \phi_{x}\right)_{x}=b(x) \phi_{t}+b(x) \tau^{(1)}(t)
$$

and the homogeneous boundary conditions

$$
\phi(0, t)=\phi(1, t)=0 \quad(-\infty<t<+\infty) .
$$

It is a consequence of (3.10) and of the variational characterization of the eigenvalue $\lambda$ that

$$
\lambda \int_{0}^{1} b \phi^{2} d x \leq \int_{0}^{1} a \phi_{x}^{2} d x=\int_{0}^{1} a u_{x}^{2} d x .
$$

Next, we use the identity

$$
\left(\psi a \phi_{x}-\phi a \psi^{\prime}\right)_{x}=\psi\left(a \phi_{x}\right)_{x}-\phi\left(a \psi^{\prime}\right)^{\prime},
$$

together with (2.18) and (3.9), and deduce that

$$
\left(\psi a \phi_{x}-\phi a \psi^{\prime}\right)_{x}=b \phi_{t}+b \tau^{(1)} \psi+\lambda b \phi \psi .
$$

Thus, on integrating with respect to $x$ we see that

$$
0=\int_{0}^{1} b \phi_{t} \psi d x+\tau^{(1)} \int_{0}^{1} b \psi d x+\lambda \int_{0}^{1} b \phi \psi d x .
$$

At this stage it is convenient to introduce a function $\sigma$ by requiring that

$$
\sigma^{(1)}=\tau-\bar{\tau}, \quad \int_{0}^{p} \sigma d t=0 .
$$

These conditions determine $\sigma$ uniquely and ensure that $\sigma$ is periodic. By virtue of Wirtinger's inequality [6, Theorem 258]

$$
\frac{2 \pi}{p}\|\sigma\| \leq\left\|\sigma^{(1)}\right\|
$$

On multiplying both sides of equation (3.12) by $\sigma$ and noting that $\tau^{(1)}=\sigma^{(2)}$, we see that

$$
\begin{aligned}
0= & \sigma \int_{0}^{1} b \phi_{t} \psi d x+\sigma \sigma^{(2)} \int_{0}^{1} b \psi d x+\lambda \sigma \int_{0}^{1} b \phi \psi d x \\
= & \frac{d}{d t}\left[\sigma \int_{0}^{1} b \phi \psi d x+\sigma \sigma^{(1)} \int_{0}^{1} b \psi d x\right] \\
& +\left(\lambda \sigma-\sigma^{(1)}\right) \int_{0}^{1} b \phi \psi d x-\left[\sigma^{(1)}\right]^{2} \int_{0}^{1} b \psi d x .
\end{aligned}
$$

Thus, if we integrate with respect to $t$ and invoke periodicity we see that

$$
\int_{0}^{1} b \psi d x \int_{0}^{p}\left[\sigma^{(1)}\right]^{2} d t=\int_{0}^{p} \int_{0}^{1}\left(\lambda \sigma-\sigma^{(1)}\right) b \phi \psi d x d t
$$


and, on dividing through by $p$, squaring both sides, and using the Cauchy-Schwarz inequality again, we have

$$
\left(\int_{0}^{1} b \psi d x\right)^{2}\left\|\sigma^{(1)}\right\|^{4} \leq \int_{0}^{1} b \psi^{2} d x \cdot \frac{1}{p} \int_{0}^{p}\left(\lambda \sigma-\sigma^{(1)}\right)^{2} d t \cdot \int_{0}^{p} \int_{0}^{1} b \phi^{2} d x d t
$$

According to the inequality $(3.11)$

$$
\frac{1}{p} \int_{0}^{p} \int_{0}^{1} b \phi^{2} d x d t \leq \frac{1}{\lambda p} \int_{0}^{p} \int_{0}^{1} a u_{x}^{2} d x d t
$$

and, according to (3.14),

$$
\begin{aligned}
\frac{1}{p} \int_{0}^{p}\left(\lambda \sigma-\sigma^{(1)}\right)^{2} d t & =\frac{1}{p} \int_{0}^{p}\left(\lambda^{2} \sigma^{2}-2 \lambda \sigma \sigma^{(1)}+\left[\sigma^{(1)}\right]^{2}\right) d t \\
& =\lambda^{2}\|\sigma\|^{2}+\left\|\sigma^{(1)}\right\|^{2} \\
& \leq\left(1+\frac{\lambda^{2} p^{2}}{4 \pi^{2}}\right)\left\|\sigma^{(1)}\right\|^{2}
\end{aligned}
$$

On combining (3.15) with (3.16) and (3.17), and cancelling a factor $\left\|\sigma^{(1)}\right\|^{2}$, we see that

$$
\left(\int_{0}^{1} b \psi d x\right)^{2}\left\|\sigma^{(1)}\right\|^{2} \leq \int_{0}^{1} b \psi^{2} d x\left(1+\frac{\lambda^{2} p^{2}}{4 \pi^{2}}\right) \cdot \frac{1}{\lambda p} \int_{0}^{p} \int_{0}^{1} a u_{x}^{2} d x d t .
$$

Since $\sigma^{(1)}=\tau-\bar{\tau}$ this proves (3.7) and so the poof of Theorem 2 is complete.

\section{REFERENCES}

[1] J. Kestin (ed.), The second law of thermodynamics, Benchmark Papers on Energy/5, Dowden, Hutchinson, and Ross, Stroudsburg, Pa., 1976

[2] C. Truesdell, The Tragicomical History of Thermodynamics 1822-1854, Springer-Verlag, New York, 1980

[3] J. Serrin, Conceptual analysis of the classical second law of thermodynamics, Arch. Rational Mech. Anal. 70, 355-371 (1979)

[4] W. A. Day, Global mean value theorems in thermodynamics, Arch. Rational Mech. Anal. 70, 181-188 (1979)

[5] _ A A commentary on thermodynamics, Springer Tracts in Natural Philosophy, vol. 32, SpringerVerlag, New York, 1988

[6] G. H. Hardy, J. E. Littlewood, and G. Pólya, Inequalities, 2nd ed., Cambridge University Press, Cambridge, 1952 